\title{
Encadrement Parental Et Developpement Emotionnel Des Adolescents Dans La Ville De Maroua, Cameroun
}

\author{
Galy Mohamadou
}

Ecole Normale Superieure, Université De Maroua, Cameroun

Doi:10.19044/esj.2018.v14n26p320 URL:http://dx.doi.org/10.19044/esj.2018.v14n26p320

\begin{abstract}
This study examines the correlation between parental supervision and emotional development among adolescents in the city of Maroua. A sample of two hundred and fifty (250) adolescents aged 11 to 19 has been selected through a simple random sampling selection. These data were subjected to the linear simple regression test. The results show that there is a correlation between parental supervision and the emotional development of adolescents. After analyzing the data collected, the hypotheses formulated on the different parental styles are: the authoritarian style (4.3\%), the democratic style $(3.6 \%)$, the permissive style $(48 \%)$ and the careless style $(4.7 \%)$, which states that parental supervision impacts on emotional development, have been confirmed. In the light of this study, it is established that what the adolescent experiences in his emotional development in the family context affects him decisively to be transposed to school and produce negative or positive effects depending on the case.
\end{abstract}

Keywords: Parenting, emotional development, adolescent, family, parenting style.

\section{Résumé}

La présente étude examine la corrélation qui existe entre l'encadrement parental et le développement émotionnel chez les adolescents de la ville de Maroua. Un échantillon de deux cents cinquante (250) adolescents de 11 à 19 ans a été sélectionné par le biais d'un échantillonnage aléatoire simple. Les données collectées ont été soumises au test de régression linéaire simple. Les résultats montrent qu'il existe une corrélation entre l'encadrement parental et le développement émotionnel des adolescents. L'analyses des données recueillies, les hypothèses formulées sur les différents styles parentaux que sont : le style autoritaire $(4,3 \%)$, le style démocratique $(3,6 \%)$, le style permissif (48\%) et le style négligent $(4,7 \%)$, qui stipulent que l'encadrement parental impacte sur le développement émotionnel, ont été 
confirmées. A la lumière de cette étude, il est établi que, ce que vit l'adolescent lors de son développement émotionnel dans le cadre familial, l'affecte de manière décisive pour être transposé en milieu scolaire et par conséquent produit des effets négatifs ou positifs selon les cas.

Mots-clés : Encadrement, développement émotionnel, adolescent, cadre familial, style parental.

\section{Introduction}

L'éducation est une entreprise temporelle qui s'inscrit dans le temps et dans l'espace de façon continue. Définir l'éducation peut paraître, à première vue, comme une entreprise sans grande importance, tellement le mot est d'usage courant et que la plupart des traités de pédagogie et même des ouvrages généraux en livrent la signification. Mais si tout le monde parle de l'éducation, l'accord sur le sens du concept ne fait pas toujours l'unanimité. Pour les uns, l'éducation est l'action par laquelle on fait sortir l'être humain de l'état d'enfance pour le conduire à l'état adulte (Nadel, Gauducheau, Cuisinier et Coutelet, 2004). Pour les autres, l'éducation est une action d'éveil de ce qui se trouve déjà dans l'enfant. Si le sujet de l'éducation est l'enfant, l'éducateur est celui qui l'accompagne vers la sagesse et celui-ci doit nécessairement être un ainé éduqué (Lehalle et Mellier, 2013).

La famille occupe une place de choix en tant qu'agent éducatif naturel qui s'occupe de l'éducation de la première enfance en favorisant le développement intégral de l'individu. C'est en son sein que s'effectuent les expériences émotionnelles et affectives, gratifiantes et frustrantes de l'enfant. Pourtois et Desmet (2000), estiment que «la famille est un champ relationnel ». C'est dire que le milieu familial qui est le premier référentiel de l'enfant offre à ce dernier une sorte d'énergie vitale indispensable à toute activité en relation avec son entourage. Il s'agit surtout de la gestion des affects et des émotions dans la famille. A ce sujet, Maslow (1970) parle des besoins d'appartenance lorsqu'il évoque la théorie de la hiérarchisation des besoins. Les parents doivent beaucoup veiller sur l'enfance et l'adolescence qui constituent les périodes d'âge d'étude et du développement de l'individu les plus importantes. La participation parentale au développement émotionnel des adolescents s'avère nécessaire.

Au sein d'une famille, chaque parent procède à l'encadrement de sa progéniture selon un style approprié. Ainsi dans la littérature, on trouve différents styles parentaux qui tous concourent au bien-être développemental des enfants. En effet Diana Baumrind $(1971,1973)$ fut la première à développer trois styles parentaux que sont le style autoritaire, le style démocratique et le style permissif. Maccoby et Martin (1983), ont ensuite ajouté un autre, qu'est le style négligent ou désengagé. Ces différents styles 
peuvent avoir une influence sur le développement émotionnel des adolescents. Dans la présente recherche, il est question de l'encadrement parental et son impact sur le développement émotionnel des adolescents. Il convient de préciser que l'observation directe ayant suscité le questionnement sur ce sujet s'est faite dans les environnements familiaux, scolaires et sociaux et il est apparu que les adolescents d'un certain âge semblent être très agités. Ils manifestent des comportements étranges comme la surestimation, la prise de risques, les oppositions aux exigences parentales et scolaires. Cette observation nous amène à suivre de près ces adolescents afin d'explorer certains aspects du développement émotionnel en l'occurrence l'expression, la compréhension et la régulation des émotions.

L'ensemble de ces comportements ont une répercussion sur le développement émotionnel qui selon Lewis (2011), pendant la tendre enfance et la petite enfance est essentiel pour plusieurs aptitudes. Lorsque celui-ci est mal négocié, cela conduit l'enfant ou l'adolescent à développer un comportement déviant. Comparativement aux adolescents éprouvant des problèmes de développement émotionnel, les adolescents dont la compétence émotionnelle est plus développée sont plus susceptibles de continuer d'apprendre, d'adopter des comportements empathiques, d'exprimer des émotions appropriées dans divers contextes, d'utiliser des stratégies adaptatives afin de faire face aux émotions négatives et dérangeantes en l'occurrence la colère et la frustration.

A l'issue de ce constat, l'on s'est posé la question de savoir les raisons du dysfonctionnement du développement émotionnel chez les enfants. Ainsi la première réponse venue à l'esprit n'a été rien d'autre que d'explorer le milieu familial couplé aux différents types d'encadrement parental comme l'un des facteurs contribuant au développement émotionnel des adolescents. Quels sont donc les aspects étudiés de ce développement émotionnel? Lehalle et Mellier (2013) ont exploré tous ses contours et sont arrivés à la conclusion selon laquelle, le développement émotionnel se résume aux trois aspects complémentaires à savoir : l'expression, la compréhension et la régulation des émotions. Ces auteurs définissent ainsi le développement émotionnel comme l'ensemble des études portant sur les questions relatives à l'émotion c'est-à-dire les études permettant de mieux comprendre l'émotion et comment la maitriser.

Généralement, c'est au sein de la famille que, l'enfant acquiert tous les éléments relatifs aux comportements et qu'il pourrait transférer dans d'autres sphères sociales où il sera appelé à évoluer. C'est dans ce sens que Muchielli (2008) dit : « c'est le contexte interactionnel qui imprime ses « formes » dans le psychisme.» Le développement harmonieux de l'enfant sur tous ses aspects, en l'occurrence l'aspect émotionnel semble être déterminé par la qualité de l'encadrement parental/style parental. 
Le développement émotionnel dont il est question dans cette étude a déjà fait l'objet d'investigation de plusieurs chercheurs surtout dans le cadre de la psychologie de développement. Au cœur des préoccupations, se trouve la problématique des interactions du sujet avec son entourage immédiat surtout pour ce qui concerne son développement dans la parentalité. Ainsi l'objectif est de voir si l'encadrement parental impacte véritablement le développement émotionnel des adolescents. En d'autres termes, les styles parentaux qu'adoptent les parents peuvent-ils être liés aux émotions des adolescents?

Cette question est importante par ce que le développement émotionnel d'un enfant est tributaire de son environnement familial, et plus particulièrement le type d'encadrement parental qui lui a été donné. La qualité des relations familiales en général et du type d'encadrement parental en particulier, conduiraient-ils à un bon développement émotionnel ? C'est dans cette logique que Perrenoud (2004:84) affirme que:

L'ordre, la rigueur, la propreté, la fidélité à la parole donnée, la déférence pour l'autorité, la capacité de suspendre la violence, le sens de la coopération et la solidarité, la capacité de se taire ou de prendre sur soi ne tombe pas du ciel, mais de cultures familiales et d'itinéraires individuels très diverses, si bien que certains retrouvent à l'école un univers normatif familier, alors que d'autre sont au sens propres « déboussolés », privés de repères habituels.

Les résultats de l'enquête exploratoire menée dans un lycée de la ville de Maroua, montrent que sur soixante-trois (63) adolescents, seulement dix-neuf (19) donnent satisfaction à travers leurs différents comportements, concernant l'expression du développement émotionnel, soit un pourcentage d'environ $31.16 \%$. Les quarante-quatre (44) autres élèves, ont été défavorables quant à ce qui est de l'expression de leur développement émotionnel, soit un pourcentage d'environ $68.84 \%$. Cette catégorie d'adolescents représente en quelque sorte ceux qui éprouvent des difficultés. Au regard de ces résultats, les adolescents dans certains établissements de la ville de Maroua, affichent des comportements émotifs et posent des actes qui vont à l'encontre des valeurs éthiques prônées par l'éducation. La fréquence des bagarres, les disputes, les injures, sont autant d'écarts de comportements liés au développement émotionnel à déplorer au sein des établissements scolaires. Qu'est ce qui peut bien expliquer ces écarts de comportements des adolescents? Ces attitudes à déplorer sont-elles liées à l'échec de l'encadrement parental? C'est au vu de ce constat qu'il est nécessaire de travailler sur l'encadrement parental et son impact sur le développement émotionnel des adolescents. Pour ce faire, l'hypothèse suivante a été formulée : l'encadrement parental influence le développement émotionnel des adolescents. 


\section{Méthodologie}

Cette recherche est de type exploratoire parce que nous nous sommes basés sur des expériences vécues quotidiennement par les enfants, ainsi que des réalités livresques pour élaborer les hypothèses. L'étude est abordée au travers d'une approche quantitative. Les variables « encadrements parentaux » et «développement émotionnel» bien que portant sur les qualités qui nécessitent des jugements de valeur, des appréciations sont du moins quantifiables. L'approche quantitative est adoptée car après administration du questionnaire, l'on a recueilli des informations qui sont analysées et interprétées quantitativement.

\section{Site d'étude}

L'étude est effectuée au lycée de Domayo à Maroua département de Diamaré, région de l'extrême-nord (Cameroun). Les données géographiques nous montrent que le lycée de Domayo est situé dans l'arrondissement de Maroua $1^{\text {er }}$, et occupe une superficie de 11 ha. Il est limité au Nord par la SODECOTON, au Sud par l'ETACRA, à l'Est par le mayo-tsanaga et à l'Ouest par la mairie de Maroua $1^{\mathrm{er}}$. Le lycée de Domayo constitue une sorte d'espace vert où, dans la cour on retrouve des multiples arbres plus particulièrement les nimiers. Le lycée n'est pas entouré par une clôture d'où son ouverture au mayo qui est un lieu connu pour la prise des drogues par les adolescents de la rue et d'autres adolescents non scolarisés ou délinquants. Cette position névralgique du lycée de Domayo à côté du mayo, pourrait contribuer à l'expérimentation des stupéfiants par les adolescents.

\section{Population mère}

La présente étude s'est intéressée aux jeunes adolescents de la ville de Maroua, plus précisément ceux du lycée de Domayo dont l'effectif des élèves, pour le $1^{\mathrm{er}}$ cycle est constitué de 937 élèves (soient 484 garçons et 453 filles). Le $2^{\text {nd }}$ cycle quant à lui, est constitué de 1135 élèves (soient 625 garçons et 510 filles). L'effectif général de l'établissement est alors de 2072 élèves, soit 1109 garçons et 963 filles. La population d'étude est dont constituée des élèves du premier cycle de l'enseignement secondaire. Ceci du fait que, l'étude sur le développement de l'enfant et de l'adolescent montre que la période d'adolescence correspond à celle des élèves du premier cycle.

\section{Echantillon}

Après le choix de l'établissement d'enquête, il a été impératif d'organiser la collecte des données selon les tranches d'âges requises. Ceci du fait que, les individus d'une population ne peuvent pas tous participer à une enquête. Après le sondage sur les différents âges des élèves dans les différentes classes, l'on se rend compte que l'âge varie entre 11 et 19 ans. Afin 
de mieux collecter les données, l'urgence a été de travailler avec les classes d'âge notamment [11-13ans], [14-16ans], [17-19ans].C'est auprès de ces derniers que l'on a recueilli des informations en rapport à ce qui a été observé. Ainsi, la dite tranche d'âge correspond aux adolescents qui fréquentent les classes de $6^{\text {ème }}, 5^{\text {ème }}, 4$ ème et $3^{\text {ème }}$ et la population cible est donc constituée des élèves de ces classes. Au sein de cette population, deux cent cinquante (250) élèves de ses classes ont constitué la population accessible.

\section{Instrument de collecte des données}

Le questionnaire utilisé est constitué d'une série de questions ouvertes s'adressant directement aux élèves et ont un lien direct avec les différentes hypothèses. Le choix de cet instrument se justifie par le fait qu'il est peu coûteux et il permet aux enquêtés de répondre librement. Il a permis aussi de gagner en temps parce que les données sont collectées au même moment. Ce questionnaire a été essentiellement adressé aux élèves de trois niveaux du premier cycle du lycée de Maroua-Domayo.

\section{Procédure}

Après la validation de l'instrument de collecte des données, l'enquête de terrain s'est effectuée les jours ouvrables et à l'heure des cours pour pouvoir trouver sur place les participants. Dans chaque classe choisie, il a fallu au préalable justifier le but de la recherche et expliquer brièvement le contenu du questionnaire avant de solliciter la réponse de chaque participant à qui un exemplaire était donné. Vingt-cinq à trente minutes étaient souvent consacrées à une classe en raison de la lenteur des répondants et des difficultés en lecture éprouvées par certains. Pour faciliter la tâche à ceux-là, le titulaire de la classe lisait progressivement le questionnaire en respectant l'ordre des questions afin que le répondant coche la réponse de son choix ou donne son impression dans un espace prévu pour les questions ouvertes.

\section{Résultats}

Les analyses descriptives présentent les distributions des répondants selon les âges et le genre. En ce qui concerne la variable âge des répondants, le tableau ci-dessous nous donne un aperçu sur leur répartition en fonction des âges.

Tableau 1 : Répartition des répondants selon leur tranche d'âge

\begin{tabular}{ccc}
\hline Age & Effectifs & Pourcentage \\
\hline$[11-13]$ & 47 & 18,8 \\
{$[14-16]$} & 159 & 63,6 \\
{$[17-19]$} & 44 & 17,6 \\
Total & $\mathbf{2 5 0}$ & $\mathbf{1 0 0}$ \\
\hline
\end{tabular}


Plus de la moitié des participants se situe dans la tranche d'âge 14-16 ans. Cette période de la croissance de l'enfant est particulièrement délicate parce qu'elle marque généralement la puberté, et peut constituer le meilleur moment pour évaluer les répercussions sur l'enfant, occasionnées par la nature de la relation affective vécue dans le cadre de l'intimité familiale.

Tableau 2 : Répartition des répondantspar genre

\begin{tabular}{ccc}
\hline Genre & Effectifs & Pourcentage \\
\hline Masculin & 113 & 45,2 \\
Féminin & 137 & 54,8 \\
Total & $\mathbf{2 5 0}$ & $\mathbf{1 0 0}$ \\
\hline
\end{tabular}

Le tableau 2 présente les participants selon le genre. Cent-treize (113) garçons qui font partis de l'échantillon représentent 45,2\%. Tandis que les filles, au nombre de cent trente-sept (137) représentent la majorité soit 54,8 \%.

En ce qui concerne la nature familiale, il ressort que cinquante (soit $20 \%$ ) participants vivent avec l'un des deux parents, et cent quarante-deux (soit 56,8\%) participants vivent dans une famille nucléaire, cinquante-huit (soit $23,2 \%$ ) participants vivent quant-à-eux dans une famille monoparentale conduite par un autre membre de la famille : la grand-mère, l'oncle, la tante, le grand frère, la grande sœur. La répartition des participants en fonction du niveau d'étude des parents montre que cinquante-trois (soit 21,2\%) d'entre eux, le parent a le niveau du primaire. Quatre-vingt-onze $(36,4 \%)$ parents ont le niveau du secondaire; quarante-six (représentant 18,4\%) autres ont le niveau du supérieur; et enfin, soixante (soit 24\%) participants n'ont aucun niveau d'étude. Ici, la majorité des parents ont au moins le niveau secondaire et par conséquent, les adolescents sont majoritairement inscrits dans les lycées, ce qui veut dire que, plus les parents ont un niveau d'éducation élevé, plus leurs enfants sont à l'école.

Le test de régression simple est utilisé et a permis ainsi d'établir s'il y a une relation significative entre les différentes les variables de l'étude (indépendante et dépendante).

\section{Vérification de la première hypothèse de recherche (H.R.1)}

- $\mathbf{H}_{\mathbf{a}}$ : il existe un lien significatif entre le style autoritaire et le développement émotionnel des adolescents.

- $\mathbf{H}_{0}$ : il n'existe aucun lien significatif entre le style autoritaire et le développement émotionnel des adolescents.

L'analyse de régression simple sur la première hypothèse de recherche montre que la valeur de $\mathrm{R}^{2}=, 043$ qui signifie que la relation est faible. Ce qui permet de comprendre que $4,3 \%$ de variance dans le développement émotionnel des adolescents est expliquée par le style autoritaire et que 95,7\% 
est expliqué par d'autres variables non-inclues dans le présent modèle. L'on constate que $\mathrm{F}(1,248)=11,157$ avec alpha $=0,0005<0,05$. L'hypothèse nulle est ainsi rejetée et l'hypothèse alternative acceptée. Par conséquent, la première hypothèse de recherche est confirmée, d'autant plus que, la valeur de Bêta qui est de 20,7\%, montre que le style autoritaire est positivement lié au développement émotionnel.

Nous référant au coefficient, on peut donc déduire l'équation estimé du modèle $\hat{y}=0,191 x+1,416$

\section{Vérification de la deuxième hypothèse de recherche (H.R.2)}

- $\mathbf{H}_{\mathbf{a}}$ :il existe un lien significatif entre le style démocratique et le développement émotionnel des adolescents.

- $\quad \mathbf{H}_{\mathbf{0}}$ :il n'existe aucun lien significatif entre le style démocratique et le développement émotionnel des adolescents.

L'analyse de régression simple sur la deuxième hypothèse de recherche révèle que $\mathrm{R}^{2}=, 36$ est relativement très faible et ceci veut dire que $36 \%$ de variance dans la le développement émotionnel des adolescents est expliquée par le style démocratique. Le reste de variance qui est de $64 \%$ est expliquée par d'autres variables non inclues dans le modèle.

$\mathrm{F}(1,248)=9,202$ avec alpha $=0,003<0,05$ implique donc la confirmation de la deuxième hypothèse de recherche. L'on en est davantage convaincu que le style démocratique influence positivement la régulation émotionnelle, car Bêta $=18,9 \%$.L'équation estimé du model est : $\hat{y}=0,211 x+1,481$

\section{Vérification de la troisième hypothèse de recherche (H.R.3)}

- $\mathbf{H}_{\mathbf{a}}$ : il existe un lien significatif entre le style permissif et le développement émotionnel des adolescents.

- $\mathbf{H}_{\mathbf{0}}$ : il n'existe aucun lien significatif entre le style permissif et le développement émotionnel des adolescents.

L'analyse de régression simple sur la troisième hypothèse de recherche, a permis de noter que $\mathrm{R}^{2}=0,48$ établit l'existence d'une relation relativement moyenne entre le style permissif et le développement émotionnel des adolescents. L'on comprend alors que $48 \%$ de variance dans le développement émotionnel est expliquée par le style permissif et le reste de $52 \%$ de cette variance est expliquée par d'autres prédicateurs non-inclus dans le présent modèle.

$\mathrm{F}(1,148)=12,488$, alpha $=0,005<0,05$. Parlant de la comparaison, il est admis que la valeur positive de $\mathrm{r}^{2}$ implique le lien positif entre la variable indépendante et la variable dépendante, ce qui permet de rejeter 1 ' $\mathrm{H}_{0}$ et d'accepter l' $\mathrm{H}_{\mathrm{a}}$. La troisième hypothèse de recherche de cette étude est ainsi confirmée. 
Bêta $=21,9 \%$ avec le seuil de significativité toujours inférieur à 0,05 . D'après le tableau de coefficient on peut donc déduire l'équation estimé du modèle est : $\hat{y}=0,203 x+1,395$

\section{Vérification de la quatrième hypothèse de recherche (H.R.4)}

- $\mathbf{H}_{\mathbf{a}}$ :il existe un lien significatif entre le style négligent et le développement émotionnel des adolescents.

- $\mathbf{H}_{0}$ :il n'existe aucun lien significatif entre le style négligent et le développement émotionnel des adolescents.

L'analyse de régression simple de la quatrième hypothèse de recherche, a permis de noter que $\mathrm{R}^{2}=, 047$ aussi l'existence d'une relation relativement faible entre le style négligent et le développement émotionnel des adolescents, avec la valeur de $\mathrm{R}^{2}=, 047 \%$. L'on comprend alors que $4,7 \%$ de variance dans la variable dépendante est expliquée par la variable indépendante; le reste 95,3\% est expliqué par d'autres prédicateurs non-inclus dans le présent modèle.

$\mathrm{F}(1,148)=12,142$ et que alpha $=0,001<0,05$. Parlant de la comparaison, il est admis que la valeur positive de $\mathrm{R}^{2}$ implique le lien positif entre la V.I. et la V.D., ce qui permet de rejeter l' $\mathrm{H}_{0}$ et d'accepter l' $\mathrm{H}_{\mathrm{a}} \mathrm{La}$ quatrième hypothèse de recherche de cette étude est ainsi confirmée.

La valeur de Bêta $=21,6 \%$ avec le seuil de significativité $=0,001$ toujours inférieur à 0,05 . D'après le tableau de coefficient on peut donc déduire l'équation estimé du modèle est : $\hat{y}=0,195 x+1,418$.

\section{Discussion}

Les recherches antérieures ont cherché à examiner l'impact de l'encadrement parental sur le développement des enfants et adolescents dans sa globalité. Si certaines études ont porté sur l'étude des disparités développementales selon les styles parentaux en relation avec la performance scolaire ou le développement cognitif d'une part et le développement des comportements déviants d'autre part, aucune à notre connaissance n'a appréhendé les trois aspects du développement émotionnel chez l'adolescent en focalisant sur l'expression, la compréhension et la et régulation dans la socialisation émotionnelle.

La présente étude visait à examiner le rapport entre l'encadrement parental et le développement émotionnel des adolescents. Dans un premier temps, nous avons montré que la valeur de $\mathrm{R}^{2}=, 043$ signifie qu'il existe une relation entre le style autoritaire et le développement émotionnel et l'on constate que $\mathrm{F}(1,248)=11,157$ avec alpha $=0,0005<0,05$ ce qui nous conduit à conclure qu'il que la valeur positive de $\mathrm{r}^{2}$ implique le lien positif entre la variable indépendante et la variable dépendante. Ces résultats sont congruents avec les conclusions de recherches précédentes examinant le lien entre le 
style parental autoritaire et le développement cognitif des adolescents (Maccoby et Martin, 1983).

Nous avons aussi procédé à l'analyse des modèles de régressions pour connaître l'impact du style parental démocratique sur le développement émotionnel. Les résultats de ces analyses montrent que, toutes choses étant égales par ailleurs, le style démocratique n'a pas d'impact significatif sur le développement émotionnel. Des résultats similaires avaient déjà été rapportés dans une recherche sur le bien-être des adolescents (Deslandes et Royer, 1994; Marcott, 2005; Mohamadou, 2007).

Quant au style permissif, il a été supposé que celui-ci soit lié au développement émotionnel chez les adolescents et les résultats d'analyse de régression ont montré que le style permissif explique la variance dans le développement émotionnel à $48 \%$. Ces résultats sont congruence avec les recherches antérieures (Mohamadou, 2007).

Enfin, pour ce qui est du lien entre le style négligent et le développement émotionnel, il ressort de l'analyse de régression que ce style explique le développement à $4,7 \%$.

Le style permissif a permis de voir comment il est lié au développement émotionnel de l'enfant. Le test de régression montre que $48 \%$ de variance dans le développement émotionnel est expliquée par le style permissif. Ces résultats contredisent les résultats de recherches antérieures qui montrent que le style permissif a toujours contribué au développement déviant des adolescents (Bornstein, 2002.).

Bien que les résultats de cette recherche fournissent une source d'information pertinente et aident à une meilleure compréhension du lien entre l'encadrement parental et le développement émotionnel, quelques limites méritent d'être mises en exergue.

La première a trait à la nature exploratoire de la recherche: cette étude est basée sur une sorte de constat reporté par les répondants. La seconde a trait à l'approche-une étude considérant l'aspect longitudinal des données ouvrirait alors une perspective instructive afin de pouvoir examiner les changements des comportements parentaux vis-à-vis des différents enfants. Nous savons que chaque enfant ait une personnalité qui lui est propre. Cette approche permettrait au fil des âges, d'examiner plus précisément les relations entre les variations dans l'encadrement parental en fonction de cette personnalité enfantine et son développement émotionnel.

\section{Conclusion}

Le développement émotionnel est étudié depuis des décennies, et pourtant, peu d'études font mention des typologies de ce développement émotionnel en relation avec les caractéristiques spécifiques des adolescents. Or, il s'agit d'un point essentiel pour développer des programmes de 
l'éducation parentale ou familiale efficaces répondant aux besoins des adolescents à différents stades de leur développement. En effet, une meilleure connaissance de ces paramètres permettra une compréhension plus fine de la relation entre parents et enfants. La présente étude avait pour but de voir s'il existe un rapport entre l'encadrement parental et le développement émotionnel des adolescents. Autrement dit, le développement émotionnel d'un adolescent est perçu dans son entièreté (expression émotionnel, compréhension et régulation des émotions) qui est supposé tributaire de la qualité de l'encadrement que lui ont donné ses parents. L'on a attribué pour cause des problèmes liés au développement émotionnel des enfants, le laxisme dans l'encadrement parental. Au regard des résultats obtenus, il ressort clairement que, le développement émotionnel des adolescents est fortement lié par l'encadrement parental à travers les différents styles parentaux.

Cette recherche a donné l'opportunité de faire des suggestions à l'endroit des parents qui sont les premiers éducateurs et la famille constituant l'environnement écologique immédiat et en quelque sorte le moule qui forge la personnalité de l'enfant/adolescent. C'est dire donc que, l'encadrement parental est lié considérablement au développement émotionnel de l'adolescent. Bref, il est clair que le développement humain dépend en grande partie de la nature des interactions sociales et lorsque celles-ci sont entachées des défaillances, et sont tortueuses ou frustrantes, elles répercutent négativement sur le développement de l'individu et dans le cas d'espèce celui du domaine affectif. L'individu se montre alors vulnérable devant toute situation complexe de la vie et, ne pourrait en aucun cas relever un défi quelconque (incapable de réguler ses émotions). Les résultats de cette étude interpellent donc les parents à plus de responsabilité en ce qui concerne la socialisation des enfants. Il est souhaité que d'autres recherches complémentaires puissent être menées dans le même cadre avec une autre population afin de rendre plus compréhensible le lien qui existe entre l'encadrement parental et le développement des adolescents en général donc un développement émotionnel compréhensif qui rime avec les normes éthiques. Par ailleurs la personnalité comme variable modératrice entre l'enfant et l'encadrement parental doit être prise en considération dans les recherches ultérieures.

\section{References:}

1. Baumrind, D. (1973). Parental disciplinary pattern and social competence. Children, youth and society, (9), 239-276.

2. Bornstein MH. (2002). Handbook of Parenting. ( $2^{\text {nd }}$ ed.). Mahwah, NJ: Erlbaum. 
3. Deslandes, R. \& Royer, É. (1994). Style parental, participation parentale dans le suivi scolaire et réussite scolaire. Service social, 43 (2), 63-80. doi:10.7202/706657ar.

4. Gramszlo C, Woodruff-Borden J. Emotional reactivity and executive control: A pathway of risk for the development of childhood worry.

Journal of Anxiety Disorders. 35, 35-41. PMID 26325305 DOI: 10.1016/j.janxdis.2015.07.005

5. Lehalle, H., Mellier, D. (2013). Psychologie de développement: Enfance et adolescence Cours et exercices ( $3^{\mathrm{e}}$ éd.).Psycho, sup, Paris : Dunod.

6. Lewis, M. (2011). Émotions - Synthèse. In: Tremblay RE. Macaire, F. (1993). Notre beau métier. Les classiques africains, (éd). Saint Paul, Paris.

7. Maccoby, E. et Martin, J. (1983). « Socialization in the context of the Family: Parent-child interaction. » In Handbook of Child Psychology, vol. 4. Socialization, Personality and Social Development, E.M. Hetherington and P.H. Mussen (dir.). New York: Wiley, p. 1-101.

8. Marcotte, J. Fortin, L., Cloutier, R., Royer, E. and Marcotte, D. (2005). Évolution de l'engagement parental auprès des élèves en difficulté de comportement et des élèves ordinaires au début du secondaire. Nouveaux cahiers de la recherche en education, 8 (2), 47-56. Doi:10.7202/1017528.

9. Maslow, A. (1970). Motivation and Personality, (2 éd.). New York: Harper \& Row. https://fr.wikipedia.org/wiki/Pyramide_des_besoins, consulté le 18 février 2016.

10. Mohamadou, G. (2007). Family environment, parenting styles and adolescents' well-being in Cameroon, ( $\mathrm{PhD}$ Thesis: unpublished). Universiti Putra Malaysia.

11. Mucchielli, A. (2008). Les motivations, P UF, Paris : Que-sais-je?

12. Perrenoud, P.(2004). Pédagogie différenciée, des interactions à l'action. ESF éditeur, 2, rue Maurice -Hartmann .92133 Issy -les Moulineaux Cedex.

13. Nadel, J., Frédérique, N. Béatrice Coutelet, C. B. (2004). Enfance, (4) Broche : PUF

14. Pourtois, J-P. et Desmet, H. (2000). Relation familiale et résilience. Paris : L'Harmattan.

15. Potvin, P. et Marcott, L. (2011). Colloque sur les compétences parentales. Participation et style parental en relation avec le risque de décrochage scolaire. Consulté le 03 février 2016.

16. Potvin, p., Marcott, D., Fortin, L. et Royer, E. (2001). Rapport du colloque sur Les Compétences Parentales et Développement .GriseUniversité de Sherbrooke. 